\title{
Homocysteine and asymmetric dimethylarginine concentrations in the plasma of Alzheimer's disease patients with varying degrees of dementia
}

\author{
Jolanta Dorszewska ${ }^{{ }^{*}}$, Jolanta Florczak-Wyspiańska ${ }^{2}$, Anna Oczkowska $^{1}$, Mateusz Dezor ${ }^{1}$, \\ Michał Prendecki ${ }^{1}$, Wojciech Kozubski ${ }^{2}$
}

\footnotetext{
${ }^{1}$ Laboratory of Neurobiology, Department of Neurology, Poznan University of Medical Sciences, Poznan, Poland;

*Corresponding Author: dorszewskaj@yahoo.com

${ }^{2}$ Chair and Department of Neurology, Poznan University of Medical Sciences, Poznan, Poland
}

Received 26 November 2012; revised 28 December 2012; accepted 10 January 2013

\begin{abstract}
Alzheimer's disease (AD) is accompanied by elevated levels of homocysteine (Hcy). Homocysteine may induce elevated concentration of asymmetric dimethylarginine (ADMA). Both Hcy and ADMA are the amino acids thought to represent risk factors of vascular diseases. Studies were conducted on the plasma levels of Hcy and methionine (Met), estimated by HPLC with electrochemical detection, as well as on levels of ADMA and arginine (Arg), estimated by HPLC with fluorescent detection, in the $A D$ patients with benign through to severe dementia estimated by MMSE scale and in a control group. The studies disclosed elevated levels of Hcy and ADMA in AD (Hcy, p < 0.001) as compared to controls, as well as in subjects older than $\mathbf{6 0}$ years of age (Hcy, $p<0.01)$. The AD patients with severe dementia have shown elevated levels of Hcy $(p<0.05)$ as compared to the patients with moderate dementia. The concentration of Met and Arg showed a downward trend in AD patients with severe dementia. The highest levels of ADMA have been demonstrated in AD patients in the early stages of the disease. In parallel, in AD with varying degrees of dementia and subjects older than 60 years of age a disturbed turnover was observed of Hcy to Met and of Arg to ADMA. Similarly to Hcy, ADMA seems to be a potential risk factor of $A D$ and important factor for progress of dementia.
\end{abstract}

Keywords: Homocysteine; ADMA; Dementia; Alzheimer's Disease

\section{INTRODUCTION}

Homocysteine (Hcy), a sulphuric amino acid is thought to represent a risk factor of both vascular and degenerative diseases. Its increased levels were found in $\mathrm{Alz}$ heimer's disease (AD) [1,2]. In the body, Hcy is metabolized along two metabolic pathways, by transsulfuration and remethylation. In the process of methylation Hcy is transformed to methionine (Met) that may undergo reverse transformation to Hcy with involvement of SAM (S-adenosylmethionine) and SAH (S-adenosylhomocysteine). (Check Figure 1) SAM is thought to provide the principal donor of methyl groups in numerous metabolic reactions, leading to formation of methyl derivatives. One of the products of SAM methylation is thought to be asymmetric dimethylarginine (ADMA) [3].

ADMA is an endogenous inhibitor of nitrogen oxide

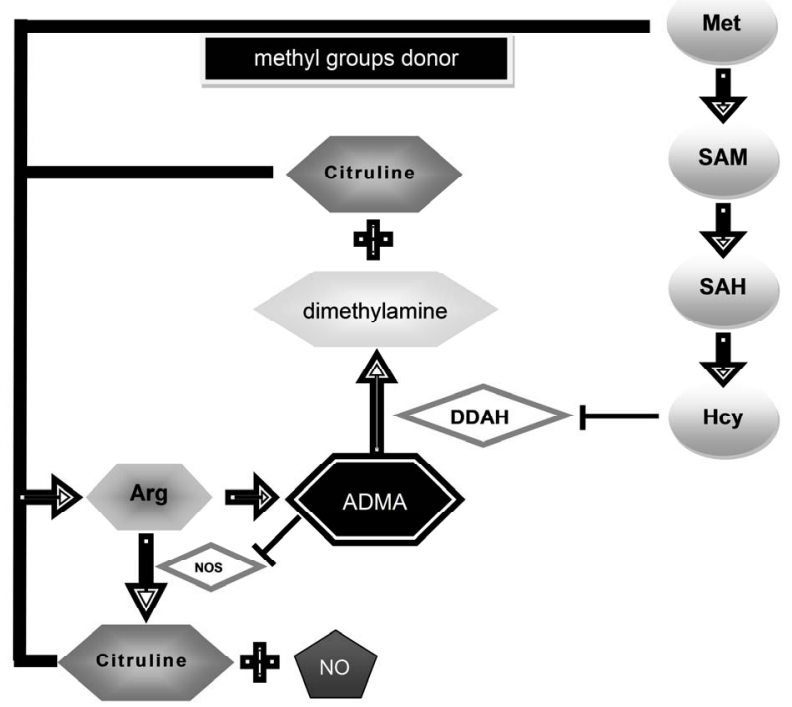

Figure 1. The roles of methionine, homocysteine and arginine in metabolism of asymmetric dimethylarginine. Met - methionine, Hcy - homocysteine, ADMA - asymmetric dimethylarginine, Arg — arginine, NO — nitric oxide, NOS - NO synthase, DDAH - dimethylarginine dimethylaminohydrolase, SAM-S-adenosylmethionine, SAH-S-adenosylhomocysteine. 
synthase (NOS) [4]. It originates from L-arginine (Arg) present in body proteins and may undergo hydrolysis to L-citruline and dimethylamine with involvement of dimethylaminohydrolase (DDAH). Homocysteine is thought to inhibit activity of DDAH [5] and might promote accumulation of ADMA that leads to a decreased production of nitrogen oxide (NO) and L-citruline from Arg with participation of NOS. NO plays an important role in control of vascular tone, in neurotransmission and in body protective mechanisms as well as in memory processes. Literature reports indicate that in $\mathrm{AD}$ the augmented activity of glia results in increased production of NO [6].

ADMA is regarded to act as a risk factor for vascular diseases [7]. Its elevated levels were demonstrated in patients with hypercholesterolemia, hypertension, chronic heart failure and atherosclerotic processes and during physiological aging [8]. ADMA is considered also to participate in pathogenesis of AD [9], most probably due to deteriorated cerebral circulation and dysfunction of vascular endothelium. However, the role of ADMA in progress of dementia in $\mathrm{AD}$ is less known.

The present study was aimed at the estimation of plasma levels of Hcy and ADMA together with Met and Arg in patients with progressive degenerative disease, $\mathrm{AD}$.

\section{MATERIAL AND METHODS}

\subsection{Patients}

The studies were conducted on 26 patients with $\mathrm{AD}$, including 16 women and 10 men aging $34-85$ years (mean age: $72.2 \pm 11.2$ years). There were $5 \mathrm{AD}$ patients with mild dementia (19\%) at the age of $42-70$ years, 12 AD patients with moderate dementia (46\%) aged $34-80$ years, and $9 \mathrm{AD}$ patients with severe dementia $(35 \%)$ aged $41-85$ years. The control group included 35 individuals, 20 women and 15 men, 22 - 76 years of age (mean age: $45.1 \pm 16.0$ years).

Patients with $\mathrm{AD}$ were diagnosed by criteria of the National Institute of Neurological and Communicative Disorders-Alzheimer's Disease and Related Disorders Association (NINCDS-ADRDA) [10]. The grade of dementia in $\mathrm{AD}$ was defined as mild (from 23 to 19 score), moderate (from 18 to 11 score) or severe (from 10 to 11 score) basing on the Mini-Mental State Examination (MMSE) scale.

Previous studies show that there are no differences in plasma Hcy levels or the progression of cognitive impairment between APOE4 carriers and noncarriers in the Polish population and refer to the papers by Religa $e t$ al. [11] and Siuda et al. [12].

In the present study, the AD patients were not divided into APOE4 carriers and noncarriers because of the small size of the cohort.
The patients were not screened for the presence of known mutations in APP or PSEN genes.

None of the control subjects had verifiable symptoms of dementia or any other neurological disorders. A Local Ethical Committee approved the study and the written consent of all patients or their caregivers was obtained.

\subsection{Analysis of Hcy and Met Concentrations}

Preparation of samples. The analyzed plasma thiol compounds (Hcy, Fluka, Germany; Met, Sigma, USA) were diluted with water at 2:1 ratio and reduced using 1\% TCEP [Tris-(2-carboxyethyl)-phosphin-hydro-chloride; Applichem, Germany] at 1:9 ratio. Subse- quently, the sample was deproteinized using $1 \mathrm{M} \mathrm{HClO}_{4}$ (at 2:1 ratio) and applied to the HPLC/EC system.

Determination of thiols concentration. The samples were injected to the HPLC system (P580A; Dionex, Germany) coupled to an electrochemical detector (CoulArray 5600; ESA, USA). The analysis was performed in Termo Hypersil BDS C18 column $(250 \mathrm{~mm} \times 4.6 \mathrm{~mm} \times$ $5 \mu \mathrm{m}$, Germany) in isocratic conditions, using the mobile phase of $0.15 \mathrm{M}$ phosphate buffer, $\mathrm{pH} 2.9$, supplemented with $12.5 \%-17 \%$ acetonitrile for estimation of Hcy and Met and $0.15 \mathrm{M}$ phosphate buffer [13].

The control of the system, data collection and processing were performed by Chromeleon software (Dionex, Germany).

\subsection{Estimation of ADMA and Arg Levels}

Preparation of samples and derivatization. Plasma and the standard, containing solution of Arg and ADMA (Sigma, USA) were diluted with water at the ratio of 1.5:1.0 and deproteinised using $8 \mathrm{M} \mathrm{HClO}_{4}$ at the ratio of 5:1. Directly before HPLC analysis the samples were subjected to derivatization in a solution containing $10 \mathrm{mg}$ o-phthalaldehyde (OPA) per $100 \mu 1$ methanol supplemented with $900 \mu \mathrm{l} 0.4 \mathrm{M}$ borate buffer ( $\mathrm{pH} \mathrm{8.5)} \mathrm{and} 5$ $\mu 1$ 2-mercaptoethanol at the ratio of 1:1 [14].

Analysis of ADMA and Arg levels. The samples were injected to the HPLC system (P580A; Dionex, Germany) coupled to a fluorescence detector (RF2000; Dionex, Germany). The analysis was performed in a Termo Hypersil BDS C18 column $(250 \mathrm{~mm} \times 4.6 \mathrm{~mm} \times 5 \mu \mathrm{m}$, Germany) in an isocratic conditions using $0.1 \mathrm{M}$ phosphate buffer, pH 6.75 with $25 \%$ methanol as the mobile phase. Arg and its methylated metabolites were measured fluorimetrically at excitation and emission wavelengths of $340 \mathrm{~nm}$ and $455 \mathrm{~nm}$, respectively.

The control of the system, data collection and processing were performed by Chromeleon software (Dionex, Germany).

Statistical analysis of results. The obtained results were analyzed using the nonparametric Mann-Whitney's 
test for unlinked variables (GraphPad, USA).

\section{RESULTS}

The plasma levels of Arg with its dimethylated metabolite, ADMA, and two thiol compounds, Hcy and its remethylation product, Met, in AD patients with different degrees of dementia as well as in the control group were studied.

In subjects older than 60 years of age the studies showed a significant increase in Hcy plasma level $(\mathrm{p}<$ 0.01 as compared to subjects between 22 and 60 years of age) accompanied by an insignificant increase in ADMA plasma level. In parallel, in this period of life, the alterations were accompanied by lowered levels of Hcy and ADMA precursors, that is: Met ( $\mathrm{p}<0.05$ as compared to individuals between 22 and 60 years of age) and Arg, thus decreased of Met/Hcy ( $p<0.01$ as compared to individuals between 22 and 60 years of age) and Arg/ ADMA ratios $(p<0.05$ as compared to individuals between 22 and 60 years of age) were observed (the data are presented in Table 1).

In patients with the diagnosed $\mathrm{AD}$ the increase of plasma Hcy levels ( $p<0.001$ as compared to the controls) was accompanied by insignificantly increased levels of circulating ADMA as compared to the controls. At the same time, in the patients with $\mathrm{AD}$, lower levels were observed of both Met $(\mathrm{p}<0.05$ as compared to the controls), and Arg expressed also by the lowered Met/ Hcy ratio ( $p<0.001$ as compared to the controls) and Arg/ADMA ratios ( $p<0.05$ as compared to the controls, see Table 2). However, these results need to be confirmed in a cohort containing age-matched control subjects and $\mathrm{AD}$ patients.

It was also indicated that in AD patients with severe dementia, reflected in MMSE scale (see Figure 2), only the increased concentration of Hcy was observed (Hcy, p $<0.05$ as compared to the patients with moderate dementia). The concentrations of the remaining analyzed amino acids manifested in general a decreasing tendency in cases of severe dementia as compared to the patients with benign or moderate symptoms (except of Arg/ ADMA ratio).

Simultaneously, in AD patients with mild dementia, Hcy level (16.2 \pm 1.9$)$ was similar to the level in controls above age $60(16.7 \pm 2.9)$. However, in AD patients with moderate dementia, Hcy level was decreased $(14.7 \pm 3.3)$. In the case of $\mathrm{AD}$ patients with severe dementia was observed an increasing trend of Hcy levels (22.4 \pm 12.0$)$. While in $\mathrm{AD}$ patients with mild, moderate and severe dementia ADMA levels were always above the level found in subjects above 60 years old and were respectively $3.2 \pm 1.3,2.7 \pm 2.0,2.8 \pm 2.3$ (see Table 1 and Figure 2).
Table 1. Homocysteine $(\mu \mathrm{M})$, methionine $(\mu \mathrm{M})$, asymmetric dimethylarginine $(\mu \mathrm{M})$ and arginine $(\mu \mathrm{M})$ concentrations in control groups.

\begin{tabular}{ccc}
\hline \multirow{2}{*}{ Parameter } & \multicolumn{2}{c}{ Age groups } \\
\cline { 2 - 3 } & $\begin{array}{c}\text { below 60 years of age } \\
(22-60 \text { years })\end{array}$ & $\begin{array}{c}\text { above 60 years of age } \\
(63-76 \text { years })\end{array}$ \\
\hline Hcy & $12.2 \pm 4.2$ & $16.7 \pm 2.9^{* *}$ \\
Met & $24.6 \pm 6.2$ & $21.0 \pm 9.7^{*}$ \\
Met/Hcy & $2.2 \pm 0.9$ & $1.3 \pm 0.6^{* *}$ \\
Arg & $82.7 \pm 24.2$ & $66.4 \pm 22.7$ \\
ADMA & $1.9 \pm 1.0$ & $2.4 \pm 0.9$ \\
Arg/ADMA & $58.8 \pm 40.6$ & $39.9 \pm 39.9^{*}$ \\
\hline
\end{tabular}

Results are expressed as mean \pm SD. Mann-Whitney test. Differences sig nificant at ${ }^{*} \mathrm{p}<0.05,{ }^{* *} \mathrm{p}<0.01$, as compared to the control below 60 years of age.

Table 2. Homocysteine $(\mu \mathrm{M})$, methionine $(\mu \mathrm{M})$, asymmetric dimethylarginine $(\mu \mathrm{M})$ and arginine $(\mu \mathrm{M})$ concentrations in the patients with $\mathrm{AD}$ and in control group.

\begin{tabular}{ccc}
\hline \multirow{2}{*}{ Parameter } & \multicolumn{2}{c}{ Investigated groups } \\
\cline { 2 - 3 } & $\begin{array}{c}\text { Controls } \\
(22-76 \text { years })\end{array}$ & $\begin{array}{c}\text { Patients with } \mathrm{AD} \\
(34-85 \text { years })\end{array}$ \\
\hline Hcy & $13.0 \pm 4.3$ & $19.3 \pm 9.9^{* * *}$ \\
Met & $24.0 \pm 6.9$ & $19.4 \pm 7.3^{*}$ \\
Met/Hcy & $2.1 \pm 0.9$ & $1.2 \pm 0.7^{* * *}$ \\
Arg & $79.7 \pm 24.4$ & $76.7 \pm 25.2$ \\
ADMA & $2.0 \pm 1.0$ & $2.8 \pm 2.1$ \\
Arg/ADMA & $55.3 \pm 40.5$ & $33.8 \pm 18.9^{*}$ \\
\hline
\end{tabular}

Results are expressed as a mean \pm SD. Mann-Whitney test. Differences significant at ${ }^{*} \mathrm{p}<0.05,{ }^{* *} \mathrm{p}<0.01,{ }^{* * *} \mathrm{p}<0.001$, as compared to the control.

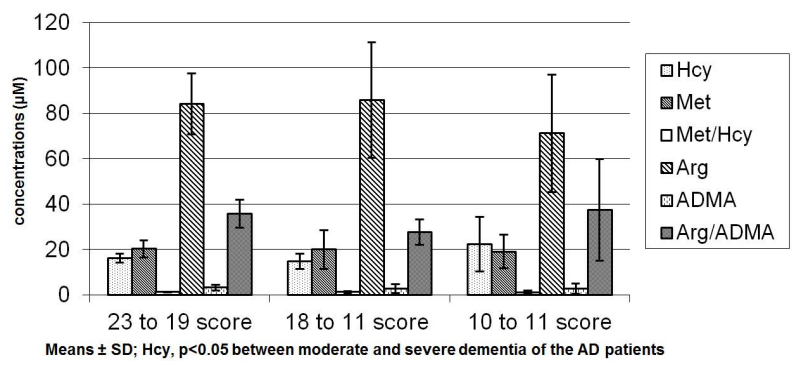

Figure 2. Hcy, Met, Arg and ADMA concentrations as related to severity of dementia (MMSE) in the patients with AD. Note: Means $\pm \mathrm{SD}$; Hcy, $\mathrm{p}<0.05$ between moderate and severe dementia of the $\mathrm{AD}$ patients.

\section{DISCUSSION}

In several degenerative diseases, including $\mathrm{AD}$, the process of pathological proteins deposition takes place. The proteins most probably induce oxydative stress 
$[15,16]$. As indicated in reports on the subject [17], also elevated levels of homocysteine (Hcy), in degenerative diseases, might be responsible for increased oxydative stress. It has been also suggested that the pro-oxidative activity of Hcy in AD most probably involves direct interaction of Hcy with NMDA receptors.

Homocysteine is thought to represent a risk factor for vascular and degenerative diseases, such as $\mathrm{AD}[1,2]$. An elevated level of Hcy in the central nervous system may lead to damage of vascular endothelium, disturbed production of nitrogen oxide and to neurotoxic effects occurring both in the senescent body and in certain neurological diseases. The conducted studies indicate that during physiological senescence increase in Hcy concentration takes place after the age of 60 years and it may result from lowered levels of Hcy metabolism cofactors due to the physiological decrease in resistance and recuperative capacities of the body. The obtained results indicate also that the increased Hcy levels may be generated by the developing degenerative disease and this pathologic process in $\mathrm{AD}$ may be linked to lack of control over activity of presenilin 1 (PS1) [18].

Hcy is thought to undergo degradation in the body along two metabolic pathways: by transsulfuration to cysteine and remethylation to Met. In present study both aging and the degenerative diseases have been shown to be accompanied by lowered levels of Met and Met to Hcy ratio, most probably due to disturbed process of Hcy remethylation. At the same time, according to a recent theory, the lowered ratio of Met to Hcy may be linked to induction of arteriosclerotic lesions by Hcy with involvement of thiolactone [19]. One of the products of Met demethylation is known to involve S-adenosylmethionine (SAM). SAM is supposed to serve as a donor of methyl groups in various methylation reactions, with participation of methyltransferases. SAM is also known to be involved in formation of asymmetric dimethylarginine (ADMA), by catalyzing reaction of arginine (Arg) methylation [3].

ADMA is regarded to act as an endogenous inhibitor of nitrogen oxide synthase (NOS). In individuals with hyperhomocysteinemia, ADMA may lead to dysfunction of vascular endothelium [20]. In parallel, Hcy is known to control ADMA concentration by blocking DDAH enzyme.

Reports of literature $[8,21,22]$ and our present study have shown that both in senescent and in AD, ADMA levels in plasma are elevated. In aging, the increased concentration of ADMA is thought to contribute to lowered accessibility of oxygen, as well as endothelial dysfunction and may be responsible for a decreased renal perfusion and increased blood pressure in senescent individuals.

On the other hand, in $\mathrm{AD}[9,21,22]$, elevated levels of ADMA may promote deterioration in cerebral circulation and dysfunction of vascular endothelium due to a decrease in NO production (leading to leukocyte aggregation, adhesion and promotion of arteriosclerotic development [23]). Disturbed function of vascular endothelium represents an important risk factor in $\mathrm{AD}$ and may promote development of the dementive disease. Also deterioration of cerebral circulation in $\mathrm{AD}$ may result in metabolic disturbances and contribute to a cognitive dysfunction and may aggravate degenerative lesions in central nervous system [24]. ADMA, thought to represent a risk factor for vascular diseases, is known to lead to arteriosclerosis, hypercholesterolemia, hypertension and strokes. All the diseases are also considered as risk factors for AD. Therefore, elevated levels of ADMA may contribute to progress of AD due to development of arteriosclerotic incidences and strokes. In parallel, the obtained results indicate that development of the dementive disease is accompanied by a tendency to decreased levels of both ADMA and its precursor, Arg, which is also responsible for generation of NO. However, there is a possibility, that the $\mathrm{AD}$ development might be associated with increased level of NO, which due to reaction of oxydation may form reactive derivatives contributing to the degenerative process [25].

This study also demonstrated that only in AD patients with severe dementia increased Hcy level is accompanied by decreased level of ADMA. As has been demonstrated in vascular disease patients, a lack of ADMA increase under high concentration of Hcy is most likely due to a concurrent mild renal insufficiency in these patients (the secretory function of the kidneys is slightly but insignificantly increased) [26].

In conclusion, this study indicated that in $\mathrm{AD}$ patients with severe dementia concentration of Met and Arg shows a downward trend. The highest levels of ADMA demonstrate $\mathrm{AD}$ patients in the early stages of the disease. In parallel, in $\mathrm{AD}$ with varying degrees of dementia and subjects older than 60 years of age a disturbed turnover observe of Hcy to Met and of Arg to ADMA. In AD patients with severe dementia Hcy control over ADMA is disturbed. It also seems that Hcy and ADMA may be potential risk factors of $\mathrm{AD}$ and important indicators of dementia progress. However, administration of L-arginine, in line with $\mathrm{B}$ vitamins to AD patients may offer a modern therapy in the neurodegenerative diseases. Analysis of Hcy and ADMA levels on a cohort of $\mathrm{AD}$ patients with varying degrees of dementia is required.

\section{REFERENCES}

[1] Seshadri, S., Beiser, A., Selhub, J., Jacques, P.F., Rosenberg, I.H., D'Agostino, R.B., Wilson, P.W. and Wolf, P.A. 
(2002) Plasma homocysteine as a risk factor for dementia and Alzheimer's disease. New England Journal of Medicine, 346, 476-483. doi:10.1056/NEJMoa011613

[2] Styczyńska, M., Strosznajder, J.B., Religa, D., Chodakowska-Zebrowska, M., Pfeffer, A., Gabryelewicz, T., Czapski, G.A., Kobryś, M., Karciauskas, G. and Barcikowska, M. (2008) Association between genetic and environmental factors and the risk of Alzheimer's disease. Folia Neuropathologica, 46, 249-254. http://goo.gl/XNLdY

[3] Gary, J.D. and Clarke, S. (1998) RNA and protein interactions modulated by protein arginine methylation. Progress in Nucleic Acid Research and Molecular Biology, 61, 65-131. doi:10.1016/S0079-6603(08)60825-9

[4] Vallance, P., Leone, A., Calver, A., Collier, J. and Moncada, S. (1992) Accumulation of an endogenous inhibitor of nitric oxide synthesis in chronic renal failure. Lancet, 339, 572-575. doi:10.1016/0140-6736(92)90865-Z

[5] Stuhlinger, M.C., Tsao, P.S., Her, J.H., Kimoto, M., Balint, R.F. and Cooke, J.P. (2001) Homocysteine impairs the nitric oxide synthase pathway: Role of asymmetric dimethylarginine. Circulation, 104, 2569-2575. doi:10.1161/hc4601.098514

[6] McGeer, P.L., Itagaki, S., Boyes, B.E. and McGeer, E.G. (1988) Reactive microglia are positive for HLA-DR in the substantia nigra of Parkinson's and Alzheimer's disease brains. Neurology, 38, 1285-1291. doi:10.1212/WNL.38.8.1285

[7] Yoo, J.H. and Lee, S.C. (2001) Elevated levels of plasma homocysteine and asymmetric dimethylarginine in elderly patients with stroke. Atherosclerosis, 158, 425-430. doi:10.1016/S0021-9150(01)00444-0

[8] Kielstein, J.T., Bode-Boger, S.M., Frolich, J.C., Ritz, E., Haller, H. and Fliser, D. (2003) Asymmetric dimethylarginine, blood pressure, and renal perfusion in elderly subjects. Circulation, 107, 1891-1895.

doi:10.1161/01.CIR.0000060496.23144.A7

[9] Selley, M.L. (2003) Increased concentration of homocysteine and asymmetric dimethylarginine and decreased concentrations of nitric oxide in the plasma of patients with Alzheimer's disease. Neurobiology of Aging, 24, 903-907. doi:10.1016/S0197-4580(03)00007-1

[10] McKhann, G.M., Knopman, D.S., Chertkow, H., Hyman, B.T., Jack, C.R. Jr., Kawas, C.H., Klunk, W.E., Koroshetz, W.J., Manly, J.J., Mayeux, R., Mohs, R.C., Morris, J.C., Rossor, M.N., Scheltens, P., Carrillo, M.C., Thies, B., Weintraub, S. and Phelps, C.H. (2011) The diagnosis of dementia due to Alzheimer's disease: Recommendations from the National Institute on Aging-Alzheimer's Association workgroups on diagnostic guidelines for Alzheimer's disease. Alzheimers and Dementia, 7, 263-269. doi:10.1016/j.jalz.2011.03.005

[11] Religa, D., Styczynska, M., Peplonska, B., Gabryelewicz, T., Pfeffer, A., Chodakowska, M., Luczywek, E., Wasiak, B., Stepien, K., Golebiowski, M., Winblad, B. and Barcikowska, M. (2003) Homocysteine, apolipoproteine E and methylenetetrahydrofolate reductase in Alzheimer's disease and mild cognitive impairment. Dementia and Geriatric Cognitive Disorders, 16, 64-70.

\section{doi: $10.1159 / 000070677$}

[12] Siuda, J., Gorzkowska, A., Patalong-Ogiewa, M., Krzystanek, E., Czech, E., Wiechuła, B., Garczorz, W., Danch, A., Jasińska-Myga, B. and Opala, G. (2009) From mild cognitive impairment to Alzheimer's disease-Influence of homocysteine, vitamin B12 and folate on cognition over time: Results from one-year follow-up. Neurologia $i$ Neu- rochirurgia Polska, 43, 321-329. http://www.termedia.pl/Czasopismo/-15/Artykul-13058

[13] Accinni, R., Bartesaghi, S., De Leo, G., Cursano, C.F., Achilli, G., Loaldi, A., Cellerino, C. and Parodi, O. (2000) Screening of homocysteine from newborn blood spots by high-performance liquid chromatography with coulometric array detection. Journal of Chromatography A, 896, 183-189. doi:10.1016/S0021-9673(00)00715-9

[14] Pi, J., Kumagai, Y., Sun, G. and Shimojo, N. (2000) Improved method for simultaneous determination of L-arginine and its mono- and dimethylated metabolites in biological samples by high-performance liquid chromatography. Journal of Chromatography B: Biomedical Sciences and Applications, 742, 199-203. doi:10.1016/S0378-4347(00)00145-6

[15] Dezor, M., Dorszewska, J., Florczak, J., Kempisty, B., Jaroszewska-Kolecka, J., Rozycka, A., Polrolniczak, A., Bugaj, R., Jagodzinski, P.P. and Kozubski, W. (2011) Expression of 8-oxoguanine DNA glycosylase 1 (OGG1) and the level of $\mathrm{p} 53$ and TNF-alpha proteins in peripheral lymphocytes of patients with Alzheimer's disease. Folia Neuropathologica, 49, 123-131. http://goo.gl/JSLhG

[16] Songin, M., Jesko, H., Czapski, G., Adamczyk, A. and Strosznajder, R.P. (2007) GSK-3beta and oxidative stress in aged brain. Role of poly(ADP-ribose) polymerase-1. Folia Neuropathologica, 45, 220-229. http://goo.gl/KaJpg

[17] Genedani, S., Rasio, G., Cortelli, P., Antonelli, F., Guidolin, D., Galantucci, M., Fuxe, K. and Agnati, L.F. (2004) Studies on homocysteine and dehydroepiandrosterone sulphate plasma levels in Alzheimer's disease patients and in Parkinson's disease patients. Neurotoxicity Research, 6, 327-332. doi:10.1007/BF03033443

[18] Fuso, A., Seminara, L., Cavallaro, R.A., D’Anselmi, F. and Scarpa, S. (2005) S-adenosylmethionine/homocysteine cycle alterations modify DNA methylation status with consequent deregulation of PS1 and BACE and betaamyloid production. Molecular and Cellular Neuroscience, 28, 195-204. doi:10.1016/j.men.2004.09.007

[19] Borowczyk, K., Tisonczyk, J. and Jakubowski, H. (2012) Metabolism and neurotoxicity of homocysteine thiolactone in mice: Protective role of bleomycin hydrolase. Amino Acids, 43, 1339-1348. doi:10.1007/s00726-011-1207-5

[20] Boger, R.H., Bode-Böger, S.M., Sydow, K., Heistad, D.D. and Lentz, S.R. (2000) Plasma concentration of asymmetric dimethylarginine, an endogenous inhibitor of nitric oxide synthase, is elevated in monkeys with hyperhomocysteinemia or hypercholesterolemia. Arteriosclerosis, Thrombosis, and Vascular Biology, 20, 1557-1564. doi:10.1161/01.ATV.20.6.1557

[21] Arlt, S., Schulze, F., Eichenlaub, M., Maas, R., Lehmbeck, 
J.T., Schwedhelm, E., Jahn, H. and Boger, R.H. (2008) Asymmetrical dimethylarginine is increased in plasma and decreased in cerebrospinal fluid of patients with Alzheimer's disease. Dementia and Geriatric Cognitive Disorders, 26, 58-64. doi:10.1159/000144026

[22] Chen, M., Jiang, P., Lu, J., Xiang, Z.H. and Jiao, B.H. (2010) The correlation of asymmetrical dimethylarginine level and oxidative stress to the onset of Alzheimer's disease. Yao Xue Xue Bao, 45, 1001-1005.

[23] Kubes, P., Suzuki, M. and Ganger, D.N. (1991) Nitric oxide: An endogenous modulator of leukocyte adhesion. Proceedings of the National Academy of Sciences USA, 88, 4651-4655. doi:10.1073/pnas.88.11.4651

[24] De la Torre, J.C. (2002) Alzheimer disease as a vascular disorder: Nosological evidence. Stroke, 33, 1152-1162. doi:10.1161/01.STR.0000014421.15948.67

[25] Su, J.H., Deng, G. and Cotman, C.W. (1997) Neuronal DNA damage precedes tangle formation and is associated with up-regulation of nitrotyrosine in Alzheimer's disease brain. Brain Research, 774, 193-199. doi:10.1016/S0006-8993(97)81703-9

[26] Jonasson, T.F., Hedner, T., Hultberg, B. and Ohlin, H. (2003) Hyperhomocysteinaemia is not associated with increased levels of asymmetric dimethylarginine in patients with ischaemic heart disease. European Journal of Clinical Investigation, 33, 543-549. doi:10.1046/j.1365-2362.2003.01184.x 